\title{
Factores que influyen en la implementación de una política pública de educación sexual integral: casos Chile y Costa Rica
}

\author{
Factors that influence the implementation of a public policy of integral sexual \\ education: Chile and Costa Rica cases \\ Emanuel Franco Mella Robles ${ }^{1}$ \\ Servicio Nacional de Menores SENAME, Chile \\ Edgar Mauricio Rebolledo Toro² \\ Universidad Católica de Temuco, Chile
}

\begin{abstract}
RESUMEN El manuscrito aborda el problema de los resultados negativos que arrojan distintos indicadores sobre sexualidad y enfermedades de transmisión sexual en Chile. La comunidad LGBTIQ, género femenino, niños, niñas y adolescentes, por ser grupos vulnerables en materia de bienestar biopsicosocial y sexual, se ven especialmente afectados y representados por este problema y sus efectos. Las causas de base más importantes son las barreras culturales, de género y de acceso a la información. Por esto, el contar con una política de educación sexual integral (ESI) basada en estándares internacionales se propone como medio para solucionar el problema desde la raíz. El artículo analiza los factores que influyen en la implementación de la ESI como asunto público, mediante la revisión de la literatura y el estudio comparativo de los casos de Costa Rica y Chile, permitiendo inferir que el rol de los movimientos sociales feminista y LGBTIQ constituye un factor relevante.
\end{abstract}

Palabras CLave Política de Educación Sexual; Feminismo; LGBTIQ.

1. Emanuel Franco Mella Robles es Magíster en Gobierno y Asuntos Públicos y Terapeuta Ocupacional. Mail: emanuel.francomella@gmail.com.

2. Edgar Mauricio Rebolledo Toro es Magíster en Gestión y Políticas Públicas y Administrador Público. Universidad Católica de Temuco. Mail: erebolledo@uct.cl. 


\begin{abstract}
The manuscript addresses the problem of negative results of different indicators on sexuality and sexually transmitted diseases in Chile. The LGBTIQ community, females, children and adolescents are vulnerable groups in terms of bio-psychosocial and sexual well-being; they are therefore particularly affected and represented by this problem and its effects. The most important root causes are cultural and gender barriers and those preventing access to information. For this reason, having a comprehensive sex education (CSE) policy based on international standards is proposed as a means of solving the problem from the roots. The article analyzes the factors that influence the implementation of CSE as a public issue, reviewing the literature and comparing the cases of Costa Rica and Chile. We infer that the role of the feminist and LGBTIQ social movements constitutes an important factor.
\end{abstract}

KEYWORDS Sex Education Policy; Feminism; LGBTIQ.

\title{
Introducción
}

El manuscrito aborda el problema de los resultados negativos que arrojan distintos indicadores sobre sexualidad y enfermedades de transmisión sexual, y la necesidad de contar con una política de educación sexual integral (ESI) en Chile. El aumento del porcentaje de personas portadoras de VIH en un 85\%, entre los años 2010 y 2018, uno de los más altos de Latinoamérica ${ }^{3}$; el inicio sexual precoz ${ }^{4}$; el bajo uso de métodos anticonceptivos; el aumento del embarazo adolescente; el aumento de las denuncias de violencia en la pareja; y las altas tasas de infecciones de transmisión sexual (ITS), por nombrar algunas; posicionan al país como uno de los más ineficaces en el manejo de este tipo de problemática en Latinoamérica ${ }^{5}$. Los resultados de la novena encuesta nacional de juventud ${ }^{6}$ confirman esta percepción, y demuestran que los índices de salud sexual; embarazo no planificado; conocimientos sobre el test de Elisa y realización del examen en jóvenes sexualmente activos; la edad de inicio de actividad sexual; el uso de preservativos; la violencia en la pareja; y la discriminación de género, indican un déficit en el abordaje integral de la sexualidad por parte de nuestra sociedad ${ }^{7}$.

3. GOLDSTEIN (2019).

4. GONZÁLEZ y RIQUELME (2018).

5. CASTRO et al. (2019); MONTERO et al. (2008).

6. INJUV (2019).

7. INJUV (2019). 
La comunidad LGBTIQ ${ }^{8}$, género femenino, los niños, niñas y adolescentes, por ser grupos vulnerables en materia de bienestar biopsicosocial y sexual, se ven especialmente afectados y representados en estos resultados ${ }^{9}$. Una de las causas más importantes es la poca información confiable y desconocimiento por parte de los jóvenes sobre la prevención y salud sexual ${ }^{10}$. De tal manera, que contar con una política de Educación Sexual Integral (ESI) que aborde el problema en todas sus dimensiones, se considera del todo urgente, pertinente y necesario para erradicar de manera eficaz esta causa ${ }^{11}$.

Chile poco ha hecho al respecto ${ }^{12}$. En un estudio sobre implementación de estrategias de educación sexual en niños y adolescentes, que considera a Latinoamérica y el Caribe, el país aparece en el último lugar del ranking ${ }^{13}$. Además, respecto de los compromisos que ha suscrito a nivel internacional sobre la materia, como el Acuerdo Interministerial del año 2008 que lo compromete con la Federación Internacional de Planificación de la Familia/Región del Hemisferio Occidental (IPPF/RHO) a generar estrategias de abordaje de la educación sexual integral a través de los ministerios de Educación y Salud, poco se ha avanzado. De hecho, se encuentra entre los países con menos porcentaje de avance y cumplimiento ${ }^{14}$.

8. LGBTIQ: L Lesbianas: mujeres con atracción sexo-afectiva a mujeres; G Gays: hombres con atracción sexo-afectiva a hombres; B Bisexuales: personas con atracción sexo-afectiva hacia hombres y mujeres; $\mathbf{T}$ Transgéneros: personas que cambian de rol de género asignado socialmente. Travestis: personas que adoptan la vestimenta e identidad del otro género de manera temporal. Transexuales: personas que se realizan alguna modificación corporal con el objetivo de asemejarse al otro sexo. I Intersexual: Se refiere a la presencia en la anatomía de una persona de órganos sexuales que corresponden a características de ambos sexos o estructuras que son difíciles de definir o resultan ambiguas desde la lógica que reconoce sólo dos sexos; Q Queer: Es un término global tomado del inglés y que define el adjetivo como «extraño» o «poco usual». Se emplea para designar a personas que no se identifican con los modelos de género binario femenino- masculino, ni con sexo hombre - mujer, así tampoco con ninguno de los mencionados anteriormente. En términos generales, rechazan clasificar a las personas por su orientación sexual o identidad de género; las y los queers buscan no encasillarse ni definirse, sino vivir en una fluidez constante, a través de la deconstrucción de los géneros socialmente impuestos. Fuente: IPS (s.f). Disponible en:: https://www. ips.gob.cl/servlet/internet/archivo/1421809741347/cartilla_div_sexual_agosto2018.pdf.

9. CLADEM (2010); GONZALEZ (2012); UNESCO (2018).

10. ALVARADO (2013); FIGUEROA (2012); OBACH et al. (2017); BEJARANO y GARCÍA (2016); UNESCO (2018).

11. UNESCO (2018); DE MARÍA et al. (2009); DIAZ (2017).

12. HUNT et al. (2015); MILES (2016); OBACH et al. (2017); VIDAL (2010).

13. HUNT et al. (2015).

14. HUNT et al. (2015). 
La sexualidad es un tema complejo de abordar, ya sea en la privacidad del hogar, escuelas y comunidad en general. Por tal razón, surgen distintas posiciones, unas a favor de una educación sexual integral abordada desde el Estado y a la sociedad en su conjunto; y otras, de carácter más conservador, que consagran la educación sexual a la esfera privada y cuestionan los límites del Estado y la familia ${ }^{15}$. Dichas posiciones se enfrentan y encuentran su expresión a través de los distintos grupos de interés, los que, con mayor o menor poder, logran hacer prevalecer sus ideas en la agenda pública y de gobierno, dificultando o facilitando la discusión pública y posterior aprobación o rechazo, según corresponda, de las políticas de $\mathrm{ESI}^{16}$.

El artículo tiene como objetivo conocer los factores que influyen en la implementación de la ESI como asunto público y abordado desde la política pública. Los objetivos específicos consisten en 1) conocer la sabiduría al respecto, estableciendo un marco teórico y una revisión de la literatura en un primer apartado; 2) contar con un análisis sobre las implicancias de una ESI según los estándares y realidades internacionales, en una segunda sección; y 3) comparar los casos de Costa Rica y Chile, a fin de inferir sobre los factores que han facilitado, el éxito del primero y el fracaso del segundo, 90\% y 39\%, respectivamente, según encuesta aplicada por la (IPPF/RHO), respecto del avance en la implementación de estrategias de educación sexual, como capítulo final ${ }^{17}$.

Para lograr estos objetivos, se hará una revisión bibliográfica especializada y un análisis comparado tomando los casos de Costa Rica y Chile, a fin de determinar diferencias y similitudes e inferir en base a factores presentes en la literatura especializada, cuáles son aquellos que podrían explicar mejores resultados en la implementación de las políticas públicas de educación sexual. El estudio es de carácter correlacional con un fin exploratorio, se determinan los casos considerando las características de cada uno, logrando comprender mejor las causas que están en las raíces del problema y sentar hipótesis para estudios posteriores.

\section{Marco teórico y revisión de la literatura}

\section{Marco conceptual - teórico}

En lo relativo al marco conceptual, entenderemos por sexualidad a aquella dimensión del ser humano que incluye conocimiento y conceptos del cuerpo, lazos afectivos, amor, sexo, género, identidad de género, orientación sexual, intimidad, placer y reproducción. Considerándolo un concepto amplio, complejo y que abarca aspectos

\footnotetext{
15. FIGUEROA (2012).

16. JIMÉNEZ (2017); RETANA (2019).

17. HUNT et al. (2015).
} 
sociales, culturales, psicológicos, espirituales, religiosos, políticos e históricos, y por lo tanto, en constante cambio y evolución ${ }^{18}$.

De igual manera, se entenderá por educación sexual como un proceso basado en un currículo que tiene como objetivo enseñar y aprender sobre los aspectos que involucran la sexualidad (psicológicos, biológicos, sociales, emocionales, etc.), para preparar a los niños, niñas y adolescentes con conocimientos, habilidades, valores y actitudes que les permitan tener una sexualidad sana, respetuosa y protegida ${ }^{19}$. Dicho proceso debe ser acorde al desarrollo de estos y basado en los derechos humanos ${ }^{20}$. Esta definición responde en particular a la definición de Educación Sexual Integral (ESI) que es la considerada como idónea según la norma internacional ya que aborda la sexualidad no sólo basada en un enfoque biologicista ${ }^{21}$.

En cuanto al marco teórico, el análisis situará a las políticas de educación sexual en el ámbito de las políticas públicas de carácter moral (PPM) ya que estas se caracterizan por manifestar distintas posiciones e ideas, tanto a favor o en contra, desde el punto de vista de los actores ${ }^{22}$, y estos representan factores determinantes en la implementación de ESI, como veremos más adelante. A su vez, vamos a señalar que la moralidad es variable entre diferentes territorios, dependiendo de la religión predominante, la cultura y el acceso a medios de comunicación. Sin embargo, los autores destacan que temáticas como el aborto, la eutanasia, el matrimonio homosexual y los métodos anticonceptivos por lo general entran en el debate moral ${ }^{23}$.

Las sociedades y Estados que se caracterizan por ser conservadores y que presentan una estrecha relación con la religión, son más resistentes a generar propuestas de políticas públicas a favor de estas temáticas. En respuesta, los grupos de interés y movimientos sociales, que cuentan cada vez con mayor participación, buscan estrategias para posicionar estas temáticas en la agenda pública, del gobierno y de las políticas públicas $^{24}$.

\section{Revisión de la literatura}

La literatura se ha enfocado en el análisis de las características que debe poseer una ESI, qué temas abordar, bajo qué enfoques trabajar, a qué población objetivo atender y en qué momento, etc. Sin embargo, no se encuentran estudios que analicen las causas

18. GONZALES (2012); HUNT et al. (2015); UNESCO (2018).

19. CLADEM (2010); UNESCO (2018).

20. CLADEM (2010); UNESCO (2015).

21. UNESCO (2015).

22. DOAN (2014).

23. Ídem.

24. Ídem. 
de por qué en algunos países sí es posible contar con políticas de educación sexual integrales según los estándares internacionales y en otros $\mathrm{no}^{25}$.

A su vez, los estudios de educación sexual han ido en aumento durante los últimos 15 años en Latinoamérica, abordando la temática desde diferentes enfoques y disciplinas, y vinculados generalmente a movimientos LGBTIQ, feminista y la crítica a la Iglesia católica ${ }^{26}$. Sin embargo, estos se han centrado fundamentalmente en los ejes de evaluación de la ejecución de política pública sobre educación sexual; comparación de los contenidos de este tipo de programas; percepción de profesores o estudiantes respecto de la educación sexual entregada; y cómo han influido los actores sociales en el surgimiento de una política pública de $\mathrm{ESI}^{27}$.

En términos metodológicos, en general, corresponden a estudios de caso que utilizan fuente de información proveniente de recursos bibliográficos, a excepción de los estudios de percepción que utilizan como recurso de obtención de datos en sujetos a través de entrevistas o encuestas ${ }^{28}$. Los enfoques teóricos se posicionan fundamentalmente desde las teorías feminista, queer y la sexualidad como un derecho individual ${ }^{29}$.

A su vez, se encontraron diversos factores que influyen en la aprobación y posterior implementación de una ESI como política pública. Por ejemplo, inclusividad, que sea de acuerdo con las necesidades de la población ${ }^{30}$, el rol que juegue la sexualidad en el control social, las relaciones de poder, la hegemonía, actores involucrados, y poder político $^{31}$. En resumen, en la literatura se señala que la ESI tiene un rol sociopolítico y que es labor del Estado propiciar un entorno que permita una implementación eficaz, legitimado y que logre incluir a todas las partes involucradas para así poder generar una cohesión social ${ }^{32}$.

25. SARABIA (2017); QUARESMA y ULLOA (2013); QUARESMA et al. (2016); MUÑOZ (2017); BAEZ (2016).

26. BAEZ (2016); MUÑOZ (2017); QUARESMA y ULLOA (2013).

27. BAEZ (2016); MUÑOZ (2017); MARTÍNEZ et al. (2013); QUARESMA y ULLOA (2013); QUARESMA et al. (2016); VIDAL (2010).

28. MARTÍNEZ et al. (2013).

29. BAEZ (2016); MARTÍNEZ et al. (2013); QUARESMA y ULLOA (2013); QUARESMA et al. (2016); VIDAL (2010).

30. DE MARÍA et al. (2009); HUNT et al. (2015); UNESCO (2015); ZAVALA (2019).

31. FOUCAULT (2007) CITADO POR DIAZ (2017); MUÑOZ (2017); RETANA (2019).

32. DE MARÍA et al. (2009); HUNT et al. (2015); UNESCO (2015); ZAVALA (2019). 
Referente a los actores, en la literatura quienes han marcado mayor presencia son, por un lado, la iglesia y los grupos conservadores, que se oponen a la educación sexual con enfoque integral, frenando su implementación en variadas ocasiones ${ }^{33}$; y por otro, las organizaciones sociales, movimiento feminista y comunidad LGBTIQ, como actores claves que se dan a la labor de movilizar la sociedad y al mundo político para que se implementen políticas de educación sexual acorde a las necesidades de la población ${ }^{34}$.

\section{Implicancias de una Educación Sexual Integral, según los estándares y realida- des internacionales}

\section{Recomendaciones de la comunidad cientifica y organizaciones no gubernamen- tales internacionales}

Estudios provenientes desde la comunidad científica y organizaciones no gubernamentales internacionales, especialistas en sexualidad, han relevado el impacto y la importancia de contar con políticas de educación sexual integrales. Dichos impactos se evidencian tanto en la conducta, conocimientos, valores y creencias de las personas cuando reciben educación sobre su sexualidad. Por tal razón, sugieren una serie de recomendaciones basadas en sus conclusiones:

- La ESI debe empezar en la niñez y continuar hasta la adultez acorde al desarrollo del niño, niña o adolescente y sus conocimientos ${ }^{35}$.

- La ESI se puede adaptar según características territoriales o cultura, pero es importante que incluya derechos de los NNA, educación sobre el cuerpo, relaciones, comportamiento y salud sexuales ${ }^{36}$.

- Requiere estrategias específicas para grupos vulnerables ${ }^{37}$.

- Debe ser inclusiva y no generar estigma, manteniendo la dignidad de las personas ${ }^{38}$.

- Debe ser integral, comprometiendo más allá del cuerpo, contenida como una construcción social, política y cultural ${ }^{39}$.

- Incorporar el género y derechos humanos ${ }^{40}$.

33. DIAZ (2017); MORGADE (2017).

34. BAEZ (2016); CLADEM (2010); MUÑOZ (2017).

35. CLADEM (2010); UNESCO (2015).

36. UNESCO (2015).

37. UNESCO (2015).

38. CÁRDENAS (2015); UNESCO (2015); UNESCO (2018).

39. CLADEM, (2010).

40. CLADEM (2010); MORGADE (2017). 
- No se compone únicamente de elementos anatómicos y fisiológicos, pues el dominio socio afectivo está también implicado ${ }^{41}$.

- Debe estar orientado bajo evidencia científica ${ }^{42}$.

- Debe ser abordado por la escuela con profesores capacitados y con el apoyo de la familia ${ }^{43}$.

Por lo tanto, según lo antes mencionado una educación debe ser integral, involucrando todas las áreas que la sexualidad abarca, respetando los derechos humanos, del niño, con enfoque de género y diversidad, a cargo del área educativa y reforzada por los padres.

\section{Educación sexual integral en el mundo y América Latina}

La Organización de las Naciones Unidas para la Educación, la Ciencia y la Cultura UNESCO realizó un estudio a nivel internacional, basado en datos sobre sexualidad y educación sexual de aproximadamente 50 países. Los resultados muestran que el $50 \%$ de las personas que tienen VIH son adolescentes y adultos jóvenes; que sólo el $34 \%$ de los jóvenes poseen conocimientos sobre prevención y transmisión del virus; y que una de cada tres mujeres en el mundo señala haber sido víctima de violencia física, psicológica o sexual por parte de parejas por motivo de género o vulneración de derecho ${ }^{44}$. A su vez, el estudio indica que el $80 \%$ de los países consultados poseen una política pública en materia de educación sexual (ES); y que la mayoría de ellos la establecen como obligatoria en la educación secundaria ${ }^{45}$.

Los países de Europa son pioneros en abordar políticas de educación sexual basadas en un enfoque integral de derechos humanos e igualdad de género, obteniendo impactos positivos para su población, como por ejemplo, la disminución de los índices de VIH y embarazos adolescentes ${ }^{46}$. A su vez, los países de Asia, reconociendo su cultura y dogmas religiosos, han logrado implementar ES bajo parámetros espirituales y en respuesta a necesidades de la población ${ }^{47}$. Sin embargo, los países de África y América Latina han sido tardíos en implementar políticas de ES, y cuando las han

41. BEJARANO y GARCÍA (2016); CALERO et al. (2017); GONZALEZ (2012); MARTÍNEZ et al. (2013).

42. ORTIZ (2017); RETANA (2019); UNESCO (2018).

43. CALERO et al. (2017); CLADEM (2010); UNESCO (2015).

44. UNESCO (2015).

45. UNESCO (2015).

46. MORGADE (2017); UNESCO (2015).

47. UNESCO (2018). 
implementado, lo han hecho bajo enfoques biologicistas ${ }^{48}$. Los efectos, se ven reflejadas en los indicadores sanitarios del problema que aborda este artículo ${ }^{49}$.

Indagando más profundamente en las raíces de dicho problema, encontramos que en AL se manifiesta una fuerte tendencia al machismo, con una relación estrecha entre el sistema sexo-género como parte intrínseca arraigada a la cultura, la política y la sociedad en general ${ }^{50}$; y con una importante tendencia a la homofobia y a la discriminación en particular ${ }^{51}$. A su vez, se caracteriza por una alta influencia de la religión, la cual se expresa en la agenda pública y en las decisiones de gobierno ${ }^{52}$.

De esta manera, podemos señalar que en las raíces del problema se encuentran barreras culturales, de género y de acceso a la información, que impiden que los jóvenes reciban una educación sexual integral y que dichas barreras explican, de alguna manera, que en Latinoamérica cada año la edad de iniciación sexual sea menor, altas tasas de embarazo adolescente, conductas sexuales de riesgo y altos índices de Infecciones de Transmisión Sexual (ITS); etc ${ }^{53}$.

Cuatro países en América Latina han empezado a tomar conciencia, y han logrado implementar políticas de ES con un enfoque integral: Argentina, Brasil, México y Costa Rica. Estos países han intentado abordar todas las áreas que la sexualidad involucra bajo normativas generales y con intervenciones a nivel de política pública, involucrando el valor a la diversidad y enfoque de género ${ }^{54}$.

\section{Antecedentes en Chile}

En Chile existe actualmente una política pública y programas de ES, sin embargo, esta es considerada aún como biologicista, no basada en el enfoque de derechos, de género y en el respeto a la diversidad ${ }^{55}$. En los años 70 se realizaron los primeros esfuerzos por implementar políticas de educación sexual en Chile. Sin embargo, estas fueron canceladas por la dictadura y reemplazadas por una educación sexual con un enfoque biológico en la asignatura de ciencias naturales ${ }^{56}$. Posteriormente, en los años 90 las

48. VIDAL (2010).

49. UNESCO $(2015 ; 2018)$.

50. CLADEM (2010).

51. VIDAL (2010).

52. MUÑOZ (2017).

53. CALERO et al. (2017); DE MARÍA et al. (2009); HUNT et al. (2015).

54. CLADEM (2010); DE MARÍA et al. (2009); HUNT et al. (2015); VIDAL (2010).

55. ALVARADO (2013); MILES (2016); GONZALES y RIQUELME (2018).

56. CESOLAA (2019). 
estrategias de ES implementadas fueron de carácter voluntarias y no lograron demostrar avances significativos ${ }^{57}$. En el año 2010, con la implementación de la ley 20.418 se incorporó la obligatoriedad de contar con educación sexual en los establecimientos educacionales, en primer y segundo año medio ${ }^{58}$.

Sin embargo, la mayoría de los temas siguen siendo abordados desde la lógica biologicista y enfocados a la prevención del embarazo adolescente, anticoncepción e ITS, y no de manera integral ${ }^{59}$. Esta situación se da fundamentalmente dadas las barreras de acceso señaladas como las raíces del problema y también porque actores de poder, como la iglesia y grupos conservadores, que han logrado imponer su postura por sobre la de los grupos progresistas ${ }^{60}$.

En conclusión, Chile presenta una tendencia similar a América Latina respecto al desarrollo de la educación sexual, ubicándolo en la situación de Europa de hace 50 años, con uno de los resultados más bajos en materia de innovación e implementación de estrategias para contrarrestar las cifras alarmantes sobre sexualidad presentadas en la problematización de este escrito ${ }^{61}$.

\section{Comparación de los casos de Chile y Costa Rica}

Para complementar la descripción general sobre el estado de la educación sexual en el mundo, América Latina y Chile, a continuación, presentamos un análisis entre Chile y Costa Rica. Este se realizará a través de un estudio de casos comparado, de carácter correlacional con un fin exploratorio ${ }^{62}$, que se llevará a cabo a través de la revisión de fuentes secundarias a fin de determinar diferencias y similitudes respecto a los factores presentes en la implementación de las políticas públicas de educación sexual.

Se determinaron los casos considerando características similares: PIB per cápita, sistema político, cultura, antigüedad de soberanía, siendo primordial en la elección los resultados obtenidos en la evaluación respecto del avance en los compromisos del Acuerdo Ministerial del año 2008 "Prevenir con Educación en Latinoamérica y el Caribe" (IPPF/RHO). En él, se encuentra que Costa Rica presenta un 9o\% de avance, mientras Chile solo un $39 \%^{63}$. No se eligieron otros casos, como Argentina, Brasil,

57. GONZÁLEZ y RIQUELME (2018).

58. GONZALES Y RIQUELME (2018).

59. CESOLAA (2019); GONZALES y RIQUELME (2018); VILLAGRA et al. (2017).

60. ALVARADO (2013).

61. HUNT et al. (2015).

62. CASTIGLIONI y FUENTES (2015).

63. HUNT et al. (2015). 
México dadas las diferencias poblacionales, territoriales y PIB per cápita. El caso de Costa Rica en todas estas características resultaba más cercano a Chile. Los casos fueron analizados en base a los hallazgos relevantes entregados por la literatura como nivel de religiosidad, presencia de organizaciones sociales feministas y/o LGBTIQ.

\section{Resultados}

El nivel de religiosidad y acción de actores conservadores; y la presencia de movimientos sociales, en específico el movimiento feminista y LGBTIQ, son relevados por la literatura como factores importantes para lograr avances o no en la implementación de políticas de educación sexual. Por esta razón, el análisis de casos se hará en base a estos factores y a las características que se deriven de los mismos. Con esto busca dar una explicación de por qué en Costa Rica se logra instaurar una educación sexual acorde a lo sugerido en los organismos internacionales y validado por la población, mientras en Chile los resultados son mucho menos positivos.

Cabe considerar que, en términos contextuales, ambos países cuentan con un PIB per cápita aproximadamente similar, sistema de gobierno presidencial, niveles de democracia equivalentes, ambos partícipes del Acuerdo Ministerial del 2008, y con presencia de organizaciones internacionales dispuestas a colaborar en el desarrollo de la educación sexual. En ambos países existen posturas que difieren la una de la otra respecto a que la educación sexual se aborde en contextos educativos de una forma integral y con enfoque de género como lo sugiere la norma internacional. Estas posturas son apoyadas por movimientos sociales progresistas, y opuestas por grupos conservadores y la iglesia ${ }^{64}$.

Respecto a la religiosidad, Latinoamérica es considerada una sociedad simpatizante con la Iglesia católica, en donde la mayoría de las personas son cristianas, y católicas $^{65}$. De esta manera Costa Rica, se presenta como un Estado confesional católico, según su constitución (1949) aún vigente ${ }^{66}$, lo que implica que todas las políticas y decisiones del país están relacionadas con la cultura y creencia cristiana siendo validada por la clase política y su población. Desde el punto de vista de las "políticas morales" se podría caracterizar como provida, profamilia y el resguardo de los valores judeocristianos ${ }^{67}$.

64. RETANA (2019).

65. ALCÁNTARA y RIVAS (2018).

66. QUESADA (2012).

67. RETANA (2019). 
En Costa Rica existe una alta relación entre la iglesia, la clase política y la elite ${ }^{68}$ lo cual ha impedido la ejecución de programas de ESI previas a la promulgada en el 2012. Esto debido a la presión que ejercieron en el gobierno los grupos conservadores y la iglesia ${ }^{69}$, incluso desde el Vaticano ${ }^{70}$. Situación similar ocurre en Chile, siendo un país categorizado como conservador y católico, en donde la iglesia busca estrategias para mantener su hegemonía y postura respectos a temas, valores y moral de la población. La iglesia es apoyada por grupos conservadores de elite y el Vaticano, logrando influir en la agenda pública y en la toma de decisiones de la clase política ${ }^{71}$.

Considerando datos estadísticos respecto a la presencia de la religión en el ámbito político y en la ciudadanía ${ }^{72}$, Costa Rica y Chile aparecen como los países más católicos de LA, presentando alta presencia de la religión en su clase política y la población en general. Respecto a los datos señalados (ver tabla 1), es importante considerar el alto porcentaje de parlamentarios católicos en ambos países, siendo un factor influyente y predisponente respecto a su postura frente a las temáticas que interfieran en los valores y la moral de la doctrina católica. Otro punto para considerar es que Costa Rica supera a Chile en todos los apartados, principalmente en la asistencia a la iglesia, en donde se puede inferir que la población en Costa Rica es católica y que además participa activamente.

Tabla 1: Presencia de la religión en el ámbito político y en la ciudadanía.

\begin{tabular}{|l|c|c|}
\hline Postulado & Chile & Costa Rica \\
\hline Parlamentarios Católicos & $78 \%$ & $80,8 \%$ \\
\hline Ciudadanos Católicos & $54,7 \%$ & $61,3 \%$ \\
\hline Asistencia iglesia 1 vez a la semana & $9,2 \%$ & $29,7 \%$ \\
\hline Asistencia a iglesia más de 1 vez a la semana & $8.1 \%$ & $13,3 \%$ \\
\hline
\end{tabular}

Fuente: Elaboración propia en base a datos de Alcántara y Rivas (2018).

68. ARGUEDAS (2010); FUENTES (2016); QUESADA (2012); RETANA (2019).

69. ARCE (2009); DÍAZ (2017).

70. MUÑOZ y ULATE (2012).

71. MORAN y VAGGIONE (2012); LAMADRID y BENITT (2019); LEHMANN (2002); QUESADA (2012).

72. ALCÁNTARA y RIVAS (2018). 
Sin embargo, tanto en Chile como en Costa Rica, la identificación religiosa ha ido disminuyendo, más aún en Chile, debido a los casos de abusos a menores ${ }^{73}$. En Costa Rica existe un panorama incierto respecto a la presencia de la religión dentro del cotidiano de la política y la población ${ }^{74}$, la iglesia aún mantiene su doctrina identitaria, y no muestra intenciones de retirarse de la agenda pública y política ${ }^{75}$. Por lo tanto, estos antecedentes darían a entender que en este factor ambos casos son similares (Costa Rica y Chile).

Por otra parte, en lo que respecta a la presencia de movimientos sociales, en Costa Rica, el movimiento feminista y LGBTIQ, han sido esenciales para el surgimiento de una $\mathrm{ESI}^{76}$, logrando posicionarla como un asunto público, en la agenda de gobierno y en la conciencia de la población, cuya opinión generalizada declara que la educación sexual debe ser impartida en los establecimientos educacionales, con el apoyo de la familia, y reconociendo el potencial beneficio que esta conlleva ${ }^{77}$.

El movimiento feminista ha estado presente en ambos países durante las últimas décadas en diferentes momentos y diferentes maneras, buscando influir en la esfera nacional. Su alianza con el movimiento LGBTIQ, se basa en objetivos afines y demandas comunes como derechos sexuales, discriminación, violencia, el patriarcado, la heteronorma y el machismo ${ }^{78}$. A su vez, han sido esenciales al relevar la necesidad de contar con educación sexual integral y el abordaje de esta en los contextos educativos. En Costa Rica, el movimiento feminista ha logrado generar instancias de participación e influenciar la agenda pública, articulando propuestas y estableciendo compromisos y acuerdos para avanzar en materia de paridad de género, salud sexual, derechos sexuales, violencia, etc ${ }^{79}$. Contando con alta participación civil y política, el movimiento feminista se ha hecho presente en el debate y desarrollo que ha tenido el país en esta materia ${ }^{80}$. La educación sexual integral se ha abordado entre este movimiento y la comunidad LGBTIQ, con el apoyo de ONG nacionales, internacionales e instituciones académicas ${ }^{81}$.

\section{LEHMANN (2002).}

74. LATINOBARÓMETRO (2007).

75. ARGUEDAS (SF); FUENTES (2016); QUESADA (2012).

76. GONZÁLEZ (2011); PIEDRA (2015); RETANA (2019).

77. DIARIO LA NACIÓN (2012); GUTIÉRREZ (2008); MUÑOZ y ULATE (2012).

78. LUNA (2006); PIEDRA (2015); QUESADA (2012).

79. GONZÁLEZ (2011); MINSAL (2011); PIEDRA (2015).

80. PIEDRA (2015).

81. MUÑOZ y ULATE (2012). 
En Chile, los movimientos sociales, se enmarcan bajo el contexto del movimiento estudiantil, que ha sido de gran importancia en la historia del país, considerando la cantidad de personas que moviliza y el impacto que provoca en la cotidianeidad de la población, agenda pública y de gobierno, todo esto en los años 2006 y 2011 principalmente $^{82}$ y el estallido social de 2019. El movimiento feminista suma a estos movimientos sociales ${ }^{83}$, sin embargo, la educación sexual, derechos sexuales y la diversidad no han logrado ser posicionados en la agenda pública por estos. Si bien, son áreas que se abordan en el marco de debates o cuestionamientos durante las elecciones presidenciales o de algún otro cargo representativo, estos movimientos no logran que sus posiciones sean abrazadas por las principales candidatas o candidatos, y menos que una vez electos, trasciendan a un programa de gobierno ${ }^{84}$.

El movimiento feminista en Chile ha tenido un avance complejo producto de la situación sociopolítica derivada de la dictadura militar. Durante la dictadura existió un movimiento cohesionado que luchó por las libertades de la mujer y la disminución de la problemática que existían en la época ${ }^{85}$. Sin embargo, con la recuperación de la democracia, se fracturó enfrentando dos posturas en su interior. Una que apoyaba su institucionalización y otra que estaba por el trabajo autónomo, sin la influencia de la institucionalidad y del gobierno. Debido a este quiebre disminuyó su visibilidad y capacidad de influencia en las acciones de la sociedad y del Estado ${ }^{86}$. En el año 2000 se observa una nueva ola de feminismo enfocado principalmente a los derechos sexuales y reproductivos, y en la visibilización de la diversidad tales como personas trans y el lesbofeminismo ${ }^{87}$.

Sin embargo, en Chile, a diferencia de Costa Rica, no se da una relación tan estrecha entre el movimiento feminista y la comunidad LGBTIQ, y menos con la finalidad de posicionar la ESI en la agenda pública. A pesar de que, en la contingencia nacional, la temática que ha sido primordial y cuenta con alta validación por parte de la población, convocatoria y visibilidad en los medios ${ }^{88}$, estos movimientos no han presentado con mayor fuerza esta necesidad.

82. LAMADRID y BENITT (2019).

83. LAMADRID y ARMIJO (2015).

84. ÁLVAREZ et al. (2017).

85. FORSTENZER (2009).

86. LAMADRID y BENITT (2019).

87. FORSTENZER (2009); LAMADRID y BENITT (2019).

88. LAMADRID y ARMIJO (2015); LAMADRID y BENITT (2019). 
El movimiento LGBTIQ ha sido uno de los promotores de la ESI, sobre todo porque son los más afectados por el problema que aborda el manuscrito. En Costa Rica, este movimiento empezó a manifestarse en el 2005 por estas demandas, con una serie de carnavales, marchas y movilizaciones multitudinaria, y que se han mantenido y aumentado con los años ${ }^{89}$. A su vez también, las publicaciones académicas y periodísticas respecto a la comunidad LGBTIQ ${ }^{90}$ y sus problemas han ido en crecimiento, a diferencia de lo que ocurre en Chile donde no abundan publicaciones científicas sobre el tema.

El movimiento LGTBIQ en Costa Rica se caracteriza por tener un sentido sociopolítico definido, y que se expresa en las actividades que se realizan en diferentes contextos y en los estudios académicos que se publican. Esta postura ha logrado fomentar la participación de la sociedad civil en temas políticos y en el debate respecto a los derechos sexuales, discriminación, diversidad e igualdad de derechos, logrando hacer visible estas posturas ${ }^{91}$.

En contraste, este enfoque sociopolítico no se visualiza de forma tan generalizada y compartida en Chile ${ }^{92}$. Han existido casos aislados de demandas en contra del Estado por vulneración de derechos o discriminación, llegando a cortes internacionales, pero no han logrado mayor visibilidad e impacto en el movimiento o en la población general93.

Respecto a la relación que el movimiento LGBTIQ tiene con el feminista, en Costa Rica este vínculo ha sido esencial para generar impacto y problematizar las situaciones de injusticia que estos grupos sufren en común ${ }^{94}$. Esta relación no se ha visto tan estrecha en Chile, siendo escasas las instancias de participación conjunta de forma organizada y con fines políticos ${ }^{95}$. En Chile, existe una serie de organizaciones que están relacionadas con el movimiento LGBTIQ, no obstante, estas no han generado gran impacto en el cotidiano de la población, siendo el Movimiento de Liberación Homosexual (MOVILH) el que ha tenido mayor visibilidad. Esta ONG ha desarrollado estudios y un sistema de apoyo a la comunidad, no obstante, sigue teniendo un impacto menor, centralizado y enfocado a un grupo pequeño dentro de la comunidad ${ }^{96}$.

89. JIMÉNEZ (2017); JIMÉNEZ (2014).

90. JIMÉNEZ (2014).

91. JIMÉNEZ (2017); JIMÉNEZ (2014); QUESADA (2012).

92. ORELLANA (2017).

93. ÁLVAREZ et al. (2017).

94. PIEDRA (2015).

95. FORSTENZER (2009); LAMADRID y BENITT (2019).

96. ORELLANA (2017). 
Un punto importante para considerar respecto a los movimientos sociales, es la validación que estos tienen por parte de la población como por el gobierno y los grupos de elite. Esto no ha ocurrido en Chile, como sí en Costa Rica en donde el movimiento a favor de ESI ha sido validado y apoyado por variados agentes que favorecieron el surgimiento del programa de $\mathrm{ESI}^{97}$.

\section{¿Qué enseña el análisis de ambos casos?}

Ha sido favorecedor para el proceso en Costa Rica la alianza estratégica entre los movimientos feministas y LGTBIQ especialmente en la formulación de una propuesta de ESI. A su vez, la incorporación de aspectos espirituales de la sexualidad, ha facilitado el apoyo de grupos religiosos, permitiendo la implementación de este tipo de políticas públicas $^{98}$.

En Chile, ha existido una tendencia desfavorable en acudir a las demandas de la ciudadanía, lo cual también se ve reflejado en los movimientos que apoyan los derechos sexuales, reproductivos y la diversidad ${ }^{99}$. A esto se suma la baja coordinación interna en los movimientos feministas y LGTBIQ que explica la principal diferencia entre los casos comparados ${ }^{100}$.

En la tabla 2 es posible apreciar que los aspectos relacionados con la religión, no presentan diferencias significativas entre ambos países. Más aún, Costa Rica presenta mayores índices de religiosidad católica respecto de Chile, desde su Constitución hasta la identificación de la ciudadanía. Por lo tanto, se infiere que este factor no sería determinante en el éxito o fracaso en la implementación de políticas ESI.

97. JIMÉNEZ (2017); RETANA (2019).

98. RETANA (2019).

99. LAMADRID y BENITT (2019).

100. LAMADRID y BENITT (2019); ORELLANA (2017). 
Tabla 2: Resumen de los factores utilizados para el análisis de casos.

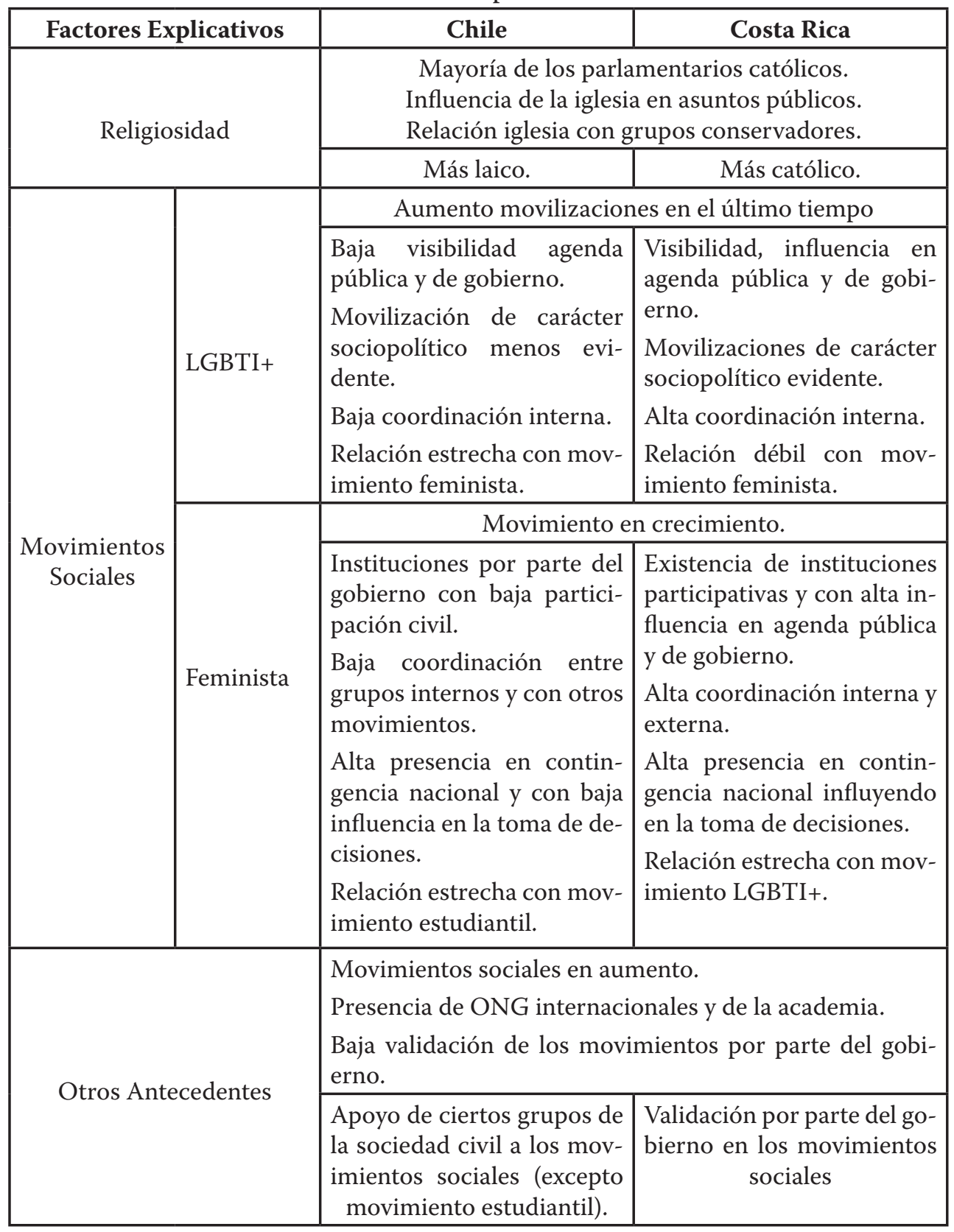

Fuente: Elaboración propia en base a datos de Minsal (2011), Muñoz \& Ulate (2012), Quesada (2012), Retana (2019), y Jiménez (2017). 
Respecto a los movimientos sociales, sí se evidencia una mayor diferencia entre ambos países. Por un lado, Costa Rica lidera en nivel de coordinación, cantidad de movimientos, organizaciones y visibilidad de los mismos, presentando una relación estrecha entre los movimientos feministas y LGBTIQ. Por otro, Chile presenta poca evolución y desarrollo de estos movimientos, y estos no han posicionado de manera determinante, o tal vez no lo han considerado importante, la ESI para así hacer frente a los problemas que en materia de salud sexual el país presenta.

\section{Conclusiones}

La educación sexual ha sido un tema de discusión en variados contextos, esto a raíz de situaciones puntuales que han formado parte de la contingencia nacional o resultados de estudios en donde Chile se posiciona dentro de los países con peores resultados. Dada la importancia y vigencia del problema, resulta pertinente preguntarse por sus causas o factores que hacen que países de "similares características" en lo concreto obtengan resultados tan diferentes. En ese marco, el artículo se propuso el objetivo de conocer los factores que influyen en la implementación de la educación sexual integral como asunto público y abordado desde la política pública.

La revisión de la literatura nos sugiere que el problema tiene distintos efectos y que estos impactan especialmente a niño, niñas, adolecentes, género femenino, y LGTBIQ. A su vez, en las raíces más profundas y causas del problema nos encontramos con barreras culturales como el machismo, una relación estrecha entre el sistema sexo-género, la política y la sociedad en general ${ }^{101}$, y con una importante tendencia a la homofobia y a la discriminación.

El rol que han jugado en el caso de Costa Rica los movimientos sociales feminista y LGBTIQ, su trabajo colaborativo y cooperación, han demostrado ser un factor relevante a la hora de implementar educación sexual integral. No así la religiosidad, sino que la espiritualidad debe estar presente en la sexualidad entendida como aquella dimensión del ser humano que incluye lazos afectivos, amor, sexo, género, identidad de género, orientación sexual, intimidad, placer y reproducción; en sentido amplio, complejo y que abarca aspectos sociales, culturales, psicológicos, espirituales, religiosos, políticos e históricos, y por lo tanto, en constante cambio y evolución.

101. CLADEM (2010). 


\section{Referencias bibliográficas}

ALCÁNTARA, Manuel y RIVAS, Cristina (2018): “América Latina: Políticos más católicos, sociedades más plurales". En Estudios de Política Exterior S.A., Madrid, Marzo de 2018. Disponible en: <https://www.politicaexterior.com/america-latina-politicos-mas-catolicos-sociedades-mas-plurales/> [Fecha de consulta: 27 de mayo de 2020 .

ALVARADO, Julia (2013): "Educación sexual preventiva en adolescentes". En Revista Contextos, UMCE, Chile, $\mathrm{N}^{\circ}$ 29, abril de 2013, pp. 25-42. Disponible en: <https:// www.umce.cl/joomlatools-files/docman-files/universidad/revistas/contextos/ N29_02.pdf> [Fecha de consulta: 27 de mayo de 2020].

ÁLVAREZ, Juan, DEL PINO, Sebastián, SAAVEDRA, María y VIAL, Tomás, (2017): "Derecho de las personas LGBTI en Chile: Una evaluación". En Informe Anual sobre Derechos Humanos en Chile 2017 (Chile, Centro de Derechos Humanos UDP). p. 472. Disponible en: < http://www.derechoshumanos.udp.cl/derechoshumanos/images/InformeAnual/2017/10-derechos\%2oLGTBI.pdf> [Fecha de consulta: 27 de mayo de 2020].

ARAVENA, Omar, CABRERAS, Claudia, CIFUENTES, Alex y VILLAGRA, Carolina (2017): "Percepción del profesorado sobre educación sexual en centros escolares de la Araucanía”. En Revista de Orientación Educacional, Universidad Católica de Temuco, año 59, $\mathrm{N}^{\circ} 31$, pp. 87-106. Disponible en: <https://dialnet.unirioja.es/servlet/articulo?codigo=6210224> [Fecha de consulta: 25 de mayo de 2020].

ARCE, Celín. (2009): "El estado y la iglesia en Costa Rica. Enfoque Jurídico”. En Revista Espiga, Universidad Estatal a Distancia, Costa Rica, $\mathrm{N}^{\circ} 18-19$, EneroDiciembre de 2009, pp. 269-304. Disponible en: < https://www.redalyc.org/ pdf/4678/467847231018.pdf $>$ [Fecha de consulta: 26 de mayo de 2020].

ARGUEDAS, Gabriela (2010): "El (aún) tortuoso camino hacia la emancipación: fundamentalismos religiosos, los derechos humanos de grupos históricamente oprimidos y la lucha por un Estado Laico en Costa Rica”. En Anuario Centro de Investigación y Estudios Políticos, Universidad de Costa Rica, Nº 1 , pp. 50-65. Disponible en: <https://revistas.ucr.ac.cr/index.php/ciep/article/view/10246/9640> [Fecha de consulta: 27 de mayo de 2020].

BÁEZ Jessica (2016): "La inclusión de la educación sexual en las políticas públicas de América Latina. Los organismos internacionales y sus formas de intervención". En Revista Latinoamericana de Educación Comparada, Sociedad Argentina de Estudios Comparados en Educación, año 7, N 9, pp. 71-86. Disponible en: < http:// www.saece.com.ar/relec/revistas/9/art5.pdf $>$ [Fecha de consulta: 26 de mayo de 2020]. 
BEJARANO, María y GARCÍA, Beatriz (2016): "La educación afectivo-sexual en España. Análisis de las leyes educativas en el periodo 1990-2016”. En Revista de Ciencias Humanas y Sociales, Universidad del Zulia, Venezuela, Año 32, N 13, pp. 756789. Disponible en: $<$ https://dialnet.unirioja.es/servlet/articulo?codigo $=5844691>$ [Fecha de consulta: 27 de mayo de 2020].

CALERO, Esmeralda, RODRÍGUEZ, Sandra y TRUMBULL, Aniosha (2017): "Abordaje de la sexualidad en la adolescencia". En Revista Humanidades Médicas, Centro de Desarrollo de las Ciencias Sociales y Humanísticas en Salud, Cuba, año 3, $\mathrm{N}^{\mathrm{o}}$ 17, pp. 577-592. Disponible en: < http://scielo.sld.cu/scielo.php?script=sci_art text\&pid=S1727-81202017000300010 $>$ [Fecha de consulta: 26 de mayo de 2020].

CASTIGLIONI, Rossana y FUENTES, Claudio (2015): "Politica comparada sobre América Latina: teorías, métodos y tópico" (Chile, Editorial Universidad Diego Portales). Disponible en: <https://www.buscalibre.cl/libro-politica-comparadasobre-america-latina-teorias-metodos-y-topicos-rossana-castiglioni-y-claudiofuentes-udp/9789563143157/p/46713993?gclid=CjoKCQjwl4V4BRDaARIsAFjATPmmba8H8kMCvrSWoXFM-c9W-oONaYNH4ZZmpFX_grtGt_-3vtE2AJL8aAh4NEALw_wcB> [Fecha de consulta: 27 de mayo de 2020].

CARDENAS, Jesús (2015): La educación sexual como estrategia de inclusión en la formación integral del adolescente. En Revista Universidad Magdalena, año 11, $\mathrm{N}^{\circ}$ 1, pp. 103-115. Disponible en: <http://revistas.unimagdalena.edu.co/index.php/ praxis/article/view/1558> [Fecha de consulta: 14 de julio de 2020].

CARRASCO, Mercedes, CASTRO, Grace, CATRIEN, Marcelo, GARCÉS, Camila, MARTICORENA, Camila y SOLAR, Francisca. (2019): "Impacto de las políticas de educación sexual en la salud sexual y reproductiva adolescente en el sur de Chile, periodo 2010-2017". En Revista Chilena de Obstetricia y Ginecología, año 84, $\mathrm{N}^{\circ}$ 1, Febrero de 2019, pp. 28-40. Disponible en: <https://scielo.conicyt.cl/scielo. php?script=sci_arttext\&pid=So717-75262019000100028> [Fecha de consulta: 27 de mayo de 2020].

CESOlAA (2019): "Curso de Educación Sexual on line de Auto Aprendizaje”. Disponible en: http://www.educacionsexual.uchile.cl/libro/\#page/1> [Fecha de consulta: 27 de mayo de 2020].

CLADEM (2010): “Género y Educación. Sistematización de Jurisprudencia de los Comités Monitores de los Derechos Humanos y del Consejo de Derechos Humanos de las Naciones Unidas". Disponible en: < https://cladem.org/publicaciones/jurisprudencia-ddhh-mujeres-salvador/> [Fecha de consulta: 27 de mayo de 2020]. 
DEMARIA, Lisa, CAMPERO, Lourdes, GALÁRRAGA, Omar, y WALKER, Dilys. (2009): "Educación sobre sexualidad y prevención del VIH: un diagnóstico para América Latina y el Caribe”. En Revista de Salud Pública de Panamá, año 29, No 6, pp. 485-493. Disponible en: <https://www.ncbi.nlm.nih.gov/pmc/articles/ PMC4720267/> [Fecha de consulta: 27 de mayo de 2020].

DÍAZ, Armando. (2017): "La contienda por los contenidos de educación sexual: repertorios discursivos y políticos utilizados por los actores en México a inicios del siglo XXI". En Debate Feminista, Universidad de Guadalajara, México, N 53, pp. 70-88. Disponible en: < http://www.debatefeminista.cieg.unam.mx/wp-content/ uploads/2017/05/articulos/DF_18.pdf> [Fecha de consulta: 27 de mayo de 2020].

DOAN, Alesha (2014): "Morality Politics". En Oxford Handbook of State and Local Government (UK, Oxford University Press), pp. 944-970. Disponible en: <https:// www.oxfordhandbooks.com/view/10.1093/oxfordhb/9780199579679.001.0001/ oxfordhb-9780199579679-e-031> [Fecha de consulta: 26 de mayo de 2020].

FIGUEROA, Essau (2012): "Política pública de educación sexual en Chile: actores y tensión entre el derecho a la información vs la libertad de elección". En Revista Chilena de Administración Pública, Universidad de Chile, $\mathrm{N}^{\circ}$ 19, pp. 105-131. Disponible en: <https://revistaeggp.uchile.cl/index.php/REGP/article/view/25861 > [Fecha de consulta: 27 de mayo de 2020].

FORSTENZER, Nicole (2009): "Ser Feminista en el Chile actual: ambigüedades y dilemas de las reivindicaciones de igualdad de género". En UMR 201 Développements et Sociétés, Université Paris I - IEDES Paris, pp. 1-12. Disponible en: < https://chilineoliberal.sciencesconf.org/conference/chili-neoliberal/pages/Forstenzer_Ser_ feminista_en_el_Chile_actual_ambiguedades_y_dilemas.pdf $>$ [Fecha de consulta: 27 de mayo de 2020].

FUENTES, Laura (2016): "Cambios en las creencias religiosas en Costa Rica”. En SIWô', Universidad de Costa Rica, año 9, Nº1, pp. 51-77. Disponible en: <https:// www.revistas.una.ac.cr/index.php/siwo/article/view/10988/13817> [Fecha de consulta: 27 de mayo de 2020].

GOLDSTEIN, Eduardo (2019): "Evolución de VIH/SIDA en Chile y países seleccionados de América Latina”. En Asesoría Técnica Parlamentaria, Biblioteca del Congreso Nacional de Chile. Disponible en: <https://www.bcn.cl/obtienearchivo?id=r epositorio/10221/27105/2/BCN_VIHSIDA_en_Chile_y_America_Latina_EG_final.pdf $>$ [Fecha de consulta: 27 de mayo de 2020]. 
GONZÁLES, Luz (2012): "Cambios en los discursos y prácticas frente a la sexualidad y la educación sexual”. En Revista Latinoamericana de Estudios de la Familia, Universidad de Caldas, Colombia, $\mathrm{N}^{\circ}$ 4, pp. 147-176. Disponible en: < http://190.15.17.25/revlatinofamilia/downloads/Rlef4_9.pdf> [Fecha de consulta: 26 de mayo de 2020].

GONZÁLEZ, Electra y RIQUELME, Roberto (2018): “Características de los Programas de Educación Sexual en Liceos de la provincia de Santiago, Chile". En Cuadernos Médicos Sociales, Colegio Médico de Chile, año 58, №2, pp. 23-30. Disponible en: <https://www.cemera.cl/publicaciones/revistas/nacionales/8_Caracteristicas_programas_educacion_sexual_liceos_provincia.pdf> [Fecha de consulta: 27 de mayo de 2020].

GONZÁLEZ, Mirta (2011): "Agenda política del movimiento de mujeres. Demandas de inicios del siglo XXI". En Revista Reflexiones, Universidad de Costa Rica, añogo, № 1, pp. 9-22. Disponible en: < https://revistas.ucr.ac.cr/index.php/reflexiones/ article/view/1469/1478> [Fecha de consulta: 26 de mayo de 2020].

GUTIÉRREZ, Blanca. (2008): “Opinión de la población de 18 años y más sobre la educación sexual en Costa Rica” (Costa Rica, Universidad Nacional, Instituto de Estudios en Población). Disponible en: <https://issuu.com/idespouna/docs/16percepcioneshacialaeducacionsexualencostarica $>$ [Fecha de consulta: 26 de mayo de 2020].

HUNT, Flor, MONTERROSAS, Erick y MIMBELA, Ricardo (2015): "Evaluación de la implementación de la declaración ministerial "Prevenir con Educación" Su cumplimiento en América Latina 2008-2015" (Estados Unidos, International Planned Parenthood Federation/Western Hemisphere Region). Disponible en: <http://incidejoven.org/wp-content/uploads/2019/11/Evaluaciones-Departamentales.pdf> [Fecha de consulta: 26 de mayo de 2020].

INJUV (2019): "Novena Encuesta Nacional de la Juventud, 2018". Disponible en: $<$ http://www.injuv.gob.cl/noticias/9encuesta [Fecha de consulta: 27 de mayo de 2020].

JIMÉNEZ, José (2014): “Temáticas en construcción: el desarrollo de los estudios LGBT en Costa Rica, 1980-2013". En Cuadernos Inter.c.a.mbio sobre Centroamérica y el Caribe, Universidad de Costa Rica, año 11, $\mathrm{N}^{\circ}$, Julio-diciembre de 2014, pp. 91-116. Disponible en: <https://revistas.ucr.ac.cr/index.php/intercambio/article/ view/16311/15813> [Fecha de consulta: 26 de mayo de 2020]. 
JIMÉNEZ, José (2017): "De lo privado a lo público: la celebración del orgullo LGBTI en Costa Rica, 2003-2016”. En Diálogos, Revista Electrónica de Historia, Universidad de Costa Rica, año18, Nº1, Enero-Junio 2017, pp. 65-9o. Disponible en: <https://www.scielo.sa.cr/scielo.php?script=sci_arttext\&pid=S1409469X2017000100065> [Fecha de consulta: 27 de mayo de 2020].

JIMÉNEZ, José (2017): “Matrimonio igualitario en Costa Rica: los orígenes del debate 1994-2006". En Revista de Ciencias Sociales, Universidad de Costa Rica, año 155, $\mathrm{N}^{\circ}$ 1, pp. 157-172. Disponible en: <https://revistas.ucr.ac.cr/index.php/sociales/article/view/30261/30233> [Fecha de consulta: 26 de mayo de 2020].

LA NACIÓN (2012): "Realidades y brechas en la educación sexual". Disponible en: <https://www.nacion.com/archivo/realidades-y-brechas-en-la-educacion-sexua 1/7SDTCFVGVVCLRL 3 JZL $_{5} \mathrm{HRTXB}_{4} \mathrm{~A} /$ story/> [Fecha de consulta: 27 de mayo de 2020].

LAMADRID, Silvia y ARMIJO, Lorena (2015): “Movimientos sociales críticos del orden de género a inicios del siglo XXI en Chile". En Congreso Latinoamericano de Teoría Social, Buenos Aires, Argentina, p. 19. Disponible en: <http://cdsa.aacademica.org/ooo-079/169.pdf> [Fecha de consulta: 27 de mayo de 2020].

LAMADRID, Silvia y BENITT, Alexandra (2019): "Cronología del movimiento feminista en Chile 2006-2016”. En Revista Estudios Feministas, Florianópolis, año 27, $\mathrm{N}^{\circ} 3$, Octubre de 2019, pp. 1-15. Disponible en: < https://www.scielo.br/pdf/ref/ v27n3/1806-9584-ref-27-03-e54709.pdf> [Fecha de consulta: 27 de mayo de 2020].

LATINOBARÓMETRO, Corporación (2007): "Informe 2007. En: Banco de datos en línea Latinobarómetro". Disponible en: <https://www.latinobarometro.org/> [Fecha de consulta: 14 de julio de 2020].

LEHMANN, Carla (2002): “¿Cuán religiosos somos los chilenos? Mapa de la religiosidad en 31 países". En Revista Estudios Públicos, Centro de Estudios Públicos, Chile, año 85, No1, pp. 21-40. Disponible en: <https://www.cepchile.cl/cep/estudios-publicos/n-61-a-la-9o/estudios-publicos-n-85/cuan-religiosos-somos-loschilenos-mapa-de-la-religiosidad-en-31-paises $>$ [Fecha de consulta: 26 de mayo de 2020].

LUNA, Lola (2006): "Mujeres y movimientos sociales". En Historia de las mujeres: España y América, España, Ediciones Cátedra, pp. 653-673. Disponible en: <http:// www.lolagluna.com/publicaciones/articulos/MujeresyMovimientos.pdf> [Fecha de consulta: 27 de mayo de 2020]. 
MARTÍNEZ, Beatriz, PUIG, Alina, RAMOS, Graciela, SERRET, Jesús y ULLOA, Ileana (2013): "Particularidades de la educación sexual en Cuba". En Revista MEDI$S A N$, Centro Provincial de Información en Ciencias Médicas, Santiago de Cuba, año 17, No 7, Marzo de 2013, pp. 2029-2040. Disponible en: <http://scielo.sld.cu/ scielo.php?script=sci_arttext\&pid=S1029-30192013000700014>. [Fecha de consulta: 27 de mayo de 2020].

MILES (2016): "Primer Informe Salud Sexual, Salud Reproductiva y Derechos Humanos en Chile" (Santiago, Chile, Corporación MILES). Disponible en: <http://www. mileschile.cl/documentos/Informe_DDSSRR_2016_Miles.pdf> [Fecha de consulta: 27 de mayo 2020].

MINSAL (2011): “Análisis de situación, propósito, enfoques, asuntos críticos y áreas de intervención de la Política de Sexualidad". En Política Nacional de Sexualidad 2010-2021 (Costa Rica, Ministerio de Salud). Disponible en: <https://www. ministeriodesalud.go.cr/index.php/biblioteca-de-archivos/sobre-el-ministerio/ politcas-y-planes-en-salud/politicas-en-salud/1039-politica-nacional-de-sexualidad-2010-2021-parte-ii/file> [Fecha de consulta: 27 de mayo 2020].

MONTERO, Adela. GONZÁLEZ, Electra y MOLINA, Temístocles (20o8): "Salud sexual y reproductiva de los y las adolescentes en Chile". En Revista de Salud Pública, Universidad de Chile, año 12, No ${ }^{1}$, pp. 42-47. Disponible en: < https://revistasaludpublica.uchile.cl/index.php/RCSP/article/view/2076>. [Fecha de consulta: 27 de mayo de 2020].

MORÁN, José y VAGGIONE, Juan (2012): “Ciencia y religión (hétero) sexuadas: el discurso científico del activismo católico conservador sobre la sexualidad en Argentina y Chile". En Contemporânea Revista de Sociologia da UFSCar, Universidade Federal de Sao Carlos, año1, No2, pp. 159-185. Disponible en: <http://www. contemporanea.ufscar.br/index.php/contemporanea/article/view/64>[Fecha de consulta: 27 de mayo de 2020].

MORGADE, Graciela (2017): “Contra el androcentrismo curricular y por una educación sexuada justa”. En Revista Internacional de Educación para la Justicia Social, Universidad Autónoma de Madrid, año 6, No 2, pp. 49-62. Disponible en: <https:// revistas.uam.es/riejs/article/view/8591> [Fecha de consulta: 27 de mayo de 2020].

MUÑOZ, María (2017): "La educación sexual en Latinoamérica: un campo de fuerzas en tensión”. En Revista Cultura del Cuidado, Universidad de Manizales, Colombia, año14, $\mathrm{N}^{\circ}{ }_{1}$, Enero-junio 2017, pp. 57-70. Disponible en: <https://dialnet.unirioja. es/servlet/articulo?codigo=6702311 $>$ [Fecha de consulta: 27 de mayo de 2020]. 
MUÑOZ, Vernor y ULATE, Carmen (2012): "El derecho humano a la educación para la afectividad y la sexualidad integral: contribuciones para una reforma educativa necesaria". En Instituto de Estudios de la Mujer, Universidad Nacional Costa Rica, pp. 12-21. Disponible en: <https://www.adc-cr.org/pdf/educacion_sexual_digital. pdf $>$ [Fecha de consulta: 26 de mayo de 2020].

OBACH, Alexandra, SADLER, Michelle y JOFRÉ, Natalia (2017): "Salud sexual y reproductiva de adolescentes en Chile: el rol de la educación sexual”. En Revista de Salud Pública, Universidad de Chile, año 19, N6, pp. 848-854. Disponible en: < http://www.scielo.org.co/pdf/rsap/v19n6/o124-oo64-rsap-19-06-848.pdf> [Fecha de consulta: 27 de mayo de 2020].

ORELLANA, Javier (2017): “Ser LGBTI en Chile y España: ¿Qué nos diferencia?”. (Instituto de la comunicación e Imagen, Universidad de Chile). Disponible en: <http:// repositorio.uchile.cl/bitstream/handle/2250/151054/TESIS-ser-lgtbi-en-chile-yespa\% 3 \%B1a.pdf? sequence $=1$ \&isAllowed $=y>$ [Fecha de consulta: 27 de mayo de 2020].

ORTIZ, Viviana (2017): "Política Pública de educación en salud sexual: de la formulación a la implantación”. En Revista Puertorriqueña de Psicología, Universidad de Puerto Rico Recinto Río Piedras, año 2, $\mathrm{N}^{\circ} 28$, Julio-diciembre de 2017, pp. 354369. Disponible en: <https://dialnet.unirioja.es/servlet/articulo? codigo=6234354> [Fecha de consulta: 25 de mayo de 2020].

PIEDRA, Nancy (2015): "La escena política de los movimientos de las mujeres en Costa Rica durante los últimos treinta años". En Revista Reflexiones, Universidad de Costa Rica, año94, No2, Mayo de 2015, pp. 65-77. Disponible en: <https://revistas. ucr.ac.cr/index.php/reflexiones/article/view/25453/25704> [Fecha de consulta: 27 de mayo de 2020].

QUARESMA, Denisse y ULLOA, Oscar (2013): "Educación sexual: estudio comparativo entre escuelas en Brasil y en Cuba". En Cuadernos de Pesquisa, Fundaçao Carlos Chagas, Brasil, año 43, No148, Abril de 2013, pp. 280-301. Disponible en: < https://www.scielo.br/pdf/cp/v43n148/14.pdf> [Fecha de consulta: 27 de mayo de 2020].

QUARESMA, Denisse, SCHMIDT, Zuleika. y CERUTTI, Márcia (2016): "Gênero, sexualidade e políticas públicas de educação". En Revista Educação, año 41, ํo1, Jan-abr 2018, pp. 49-58. Disponible en: <https://www.researchgate.net/publication/325432341_Genero_sexualidade_e_politicas_publicas_de_educacao/fulltext /5bodfd5b4585157f872234b5/Genero-sexualidade-e-politicas-publicas-de-educacao.pdf $>$ [Fecha de consulta: 27 de mayo de 2020]. 
QUESADA, Carolina (2012): "Familia y heteronormatividad: acontecimientos históricos y la doctrina sexual de la iglesia católica en Costa Rica”. En Anuario de Estudios Centroamericanos, Universidad de Costa Rica, $\mathrm{N}^{\circ} 38$, pp. 305-328. Disponible en: < https://revistas.ucr.ac.cr/index.php/anuario/article/view/1916> [Fecha de consulta: 27 de mayo de 2020].

RETANA, Camilo (2019): “Tensiones en tomo al cuerpo, el género y el deseo en los Programas de estudio de educación para la efectividad y sexualidad integral de Costa Rica”. En Revista Filosofía UIS, Universidad de Costa Rica, año1, No18, Enero-junio de 2019, pp. 130-150. Disponible en: <https://revistas.uis.edu.co/index. $\mathrm{php} /$ revistafilosofiauis/article/view/8573/9208> [Fecha de consulta: 27 de mayo de 2020].

SARABIA, Diana (2017): La educación de la salud sexual de los adolescentes en Canadá y Chile: una mirada desde las políticas públicas. En Revista Temas Sociológicos, $\mathrm{N}^{\circ}$ 1, pp. 49-96. Disponible en: <http://ediciones.ucsh.cl/ojs/index.php/TSUCSH/ article/view/193> [Fecha de consulta: 14 de julio de 2020].

UNESCO (2015): "Emerging evidence lessons and practice in comprehensive sexuality education. A global review". Disponible en: <https://www.unfpa.org/sites/default/ files/pub-pdf/CSE_Global_Review_2015.pdf> [Fecha de consulta: 27 de mayo de 2020].

UNESCO (2018): “Orientaciones técnicas internacionales sobre educación en sexualidad: un enfoque basado en la evidencia”. Disponible en: <https://unesdoc.unesco. org/ark:/48223/pfoooo265335> [Fecha de consulta: 27 de mayo de 2020].

VIDAL, Francisco (2010): "Educación sexual y diversidad en los programas educativos en América Latina”. En Revista Electrónica Diálogos Educativos, UMCE, Chile, Año 10, $\mathrm{N}^{\circ}$ 20, Octubre de 2010, pp. 77-106. Disponible en: <http://revistas.umce. $\mathrm{cl} /$ index.php/dialogoseducativos/article/view/109o/1102> [Fecha de consulta: 25 de mayo de 2020].

ZAVALA, María (2019): “De quién son los niños? Estado, familia y educación sexual en México en la década de 1930”. En Revista Signos Históricos, Universidad Autónoma Metropolitana, México, año 41, No 21, Enero-junio de 2019, pp. 154-191. Disponible en: <https://signoshistoricos.izt.uam.mx/index.php/historicos/article/ view/551/511> [Fecha de consulta: 27 de mayo de 2020]. 